\title{
Material property determination of the lining layers of a versatile helmet
}

\author{
Radek Kottner ${ }^{1, *}$, Richard Hynek ${ }^{1}$, Tomáš Mandys ${ }^{1}$, Jan Bartošek ${ }^{1}$ \\ ${ }^{1}$ NTIS - New Technologies for the Information Society, Faculty of Applied Sciences, University \\ of West Bohemia, Technicka 8, 30100 Pilsen, Czech Republic
}

\begin{abstract}
This paper deals with material property identification of a helmet lining consisting of an outer layer of an expanded polystyrene (EPS) and inner layer of an open-closed cell foam (OCCF). A combined numerical simulation and experimental testing was used for the material property identification. Compression and drop tests were performed. The ABAQUS finite element commercial code was used for numerical simulations in which the OOCF was modelled as a rate dependent viscoelastic material, while the EPS as a crushable foam. The reaction force time histories coming from the numerical simulation and the experiment have been used as a criterion for material parameter determination. After the identification of the material properties, numerical drop-tests were used to study the behaviour of a plate and a conical composite OOCF and EPS liners to decide which of them suits more for the helmet.
\end{abstract}

Keywords: helmet liner, foam, expanded polystyrene, drop test, identification

\section{Introduction}

Common property of composites and foams is their low specific weight. A utilization of these two materials in suitable combination allows the creation of lightweight impact energy absorbers where composite layers advantageously distribute impact forces into foam layers. Expanded polystyrene (EPS) is a crushable foam which, in case of compression, absorbs energy during the plastic deformation stage when the stress reaches a roughly constant plateau value (plateau region in force compression diagram) over a large percentage of total strain (typically 60-70\%)[1]. Open-closed cell foam (OCCF) is modern energy absorbing material having different viscoelastic mechanical response according to strain rate (produced e.g. by $\mathrm{D} 3 \mathrm{O} \AA$ ).

The aim of this work is the identification of parameters of material models which would sufficiently characterize the foams for the thickness determination of a versatile sport helmet lining using the finite element method (FEM) model. The versatile sport helmet having a removable chin guard and a peak is shown in Fig. 1. The helmet should be suitable for cycling, inline skating, skateboarding, horse riding, paragliding and other similar sports.

\footnotetext{
* Corresponding author: kottner@kme.zcu.cz

Reviewers: Tomasz Domański, Ladislav Écsi
} 
It has outer carbon fibre reinforced plastic (CFRP) shell with thickness of $2 \mathrm{~mm}$ (more detail description of the composite shell and its modelling can be found in [1]). The helmet lining consists of an outer layer of the EPS and inner layer of the OCCF.
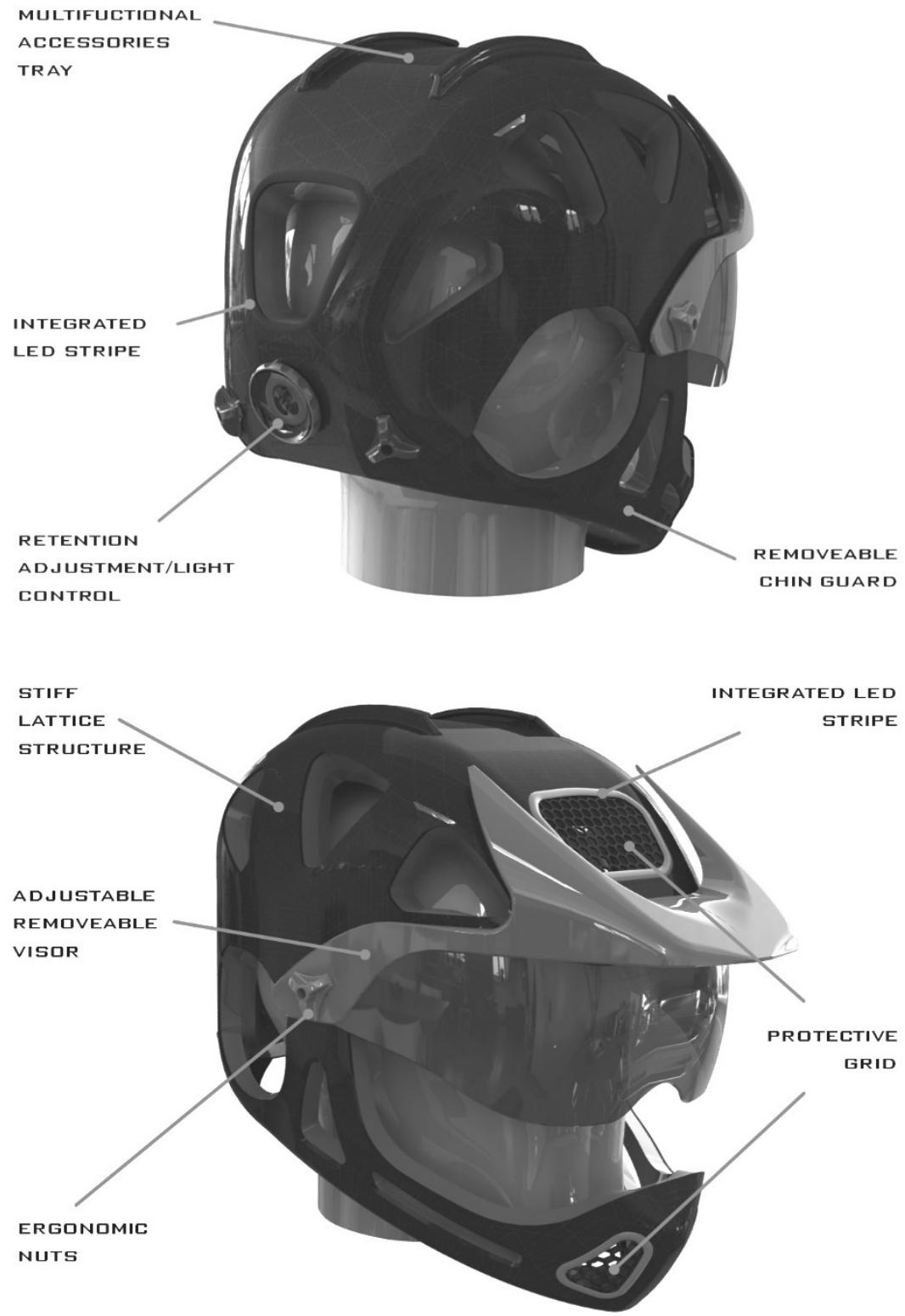

Fig. 1. Versatile sport helmet 


\section{Experiments}

Compression and drop tests were performed using a Zwick/Roell Z050 universal testing machine and a drop tower designed by the authors [3], respectively. The temperature was $23 \pm 1{ }^{\circ} \mathrm{C}$, the atmospheric moisture was $50 \pm 6 \%$. Rectangular samples of the foams measured $20 \times 20 \mathrm{~mm}$ and had a height (thickness) of 10 or $20 \mathrm{~mm}$. Densities of the EPS and OCCF were $100 \mathrm{~kg} / \mathrm{m}^{3}$ and $500 \mathrm{~kg} / \mathrm{m}^{3}$, respectively.

Two identical loading cycles were applied on the samples in the compression test using flat clamping grips. The samples were loaded and unloaded with velocity of $10 \mathrm{~mm} / \mathrm{s}$. Value of nominal strain 0.35 was prescribed as the upper limit. After reaching the upper limit of nominal strain, the testing machine maintained the strain for one minute. Zero strain was maintained also for one minute between the loading cycles.

The total weight of impactor were $246 \mathrm{~g}$ or $350 \mathrm{~g}$ in dependence of the used impactor head (spherical or flat head). The impactor was equipped by KISTLER 9712B load cell that enabled to record the time-force response (contact force) between the impactor head and a tested sample. The radius of the spherical head of the impactor was $15 \mathrm{~mm}$ (detailed description of the impactor geometry can be found in [3]), the flat head of the impactor had a square contact surface with side length of $30 \mathrm{~mm}$. Reached impact velocity $v$ varied from $1 \mathrm{~m} / \mathrm{s}$ to $5.5 \mathrm{~m} / \mathrm{s}$. The time-displacement response (impactor displacement during the impact) was investigated using the Micro-Epsilon optoNCDT laser. Data were supplemented with images from the high-speed camera OLYMPUS i-SPEED 2 (frame rate $2000 \mathrm{fps})$.

\section{Numerical simulations}

The tests were simulated in the FEM system ABAQUS. The crushable foam material model was used for the EPS tests simulations. In this model, the contribution of the mean stress on the yield function is realised through a material parameter known as a shape factor $[4,5]$. It defines the aspect ratio of the elliptical stress. The yield function $Y$ is given by

$$
Y=\sqrt{q^{2}+\alpha^{2} p^{2}}-B=0,
$$

where $q$ is the von Mises equivalent stress and $p$ is the mean stress. $B$ defines the size of the yield ellipse and is given by

$$
B=\alpha p_{c}=\sigma_{c} \sqrt{1+(\alpha / 3)^{2}},
$$

where $p_{c}$ is the yield strength in hydrostatic compression. $\sigma_{c}$ is the absolute value of the yield strength in uniaxial compression.

$$
\alpha=\frac{3 k}{\sqrt{9-k^{2}}}
$$

is the shape factor of the yield surface and $k$ is the ratio of initial yield stress in uniaxial compression and initial yield stress in hydrostatic compression.

The Prony series were used to describe viscoelastic behaviour of the OCCF. The relaxation function was assumed in the normalized series expansion [6]

$$
g_{\mathrm{R}}(t)=\frac{G_{\mathrm{R}}(t)}{G_{0}}=1-\sum_{i=1}^{N} g_{i}\left(1-e^{-t / \tau_{i}}\right),
$$

where $G_{\mathrm{R}}$ is the stress relaxation shear modulus and $G_{0}$ is the instantaneous shear modulus. $N, g_{i}$, and $\tau_{i}, i=1,2, \ldots, N$, are material parameters. Since long-term Young's 
modulus $E_{\mathrm{OCCF}}$ was identified, the instantaneous Young's and shear moduli can be determined from

$$
G_{\infty}=G_{0}\left(1-\sum_{i=1}^{N} g_{i}\right)
$$

and

$$
E_{\mathrm{OCCF}}=2 G_{\infty}\left(1+v_{\mathrm{OCCF}}\right),
$$

where $v_{\mathrm{OCCF}}$ is the Poisson's ratio of the OCCF.

The finite-strain forms of the models were considered. Parameters of the material models were identified based on comparison of data from the tests and the numerical simulations using an optimization algorithm in optiSLang software. Since the thickness of the helmet lining can be determined using numerical simulations of helmet drop tests according to standards ČSN EN 1078 (Fig. 2) and ČSN EN 1385, the emphasis was placed on the fitting of the drop tests. Moreover, the OCCF parameters were identified separately for four different impact velocities of the flat impactor.
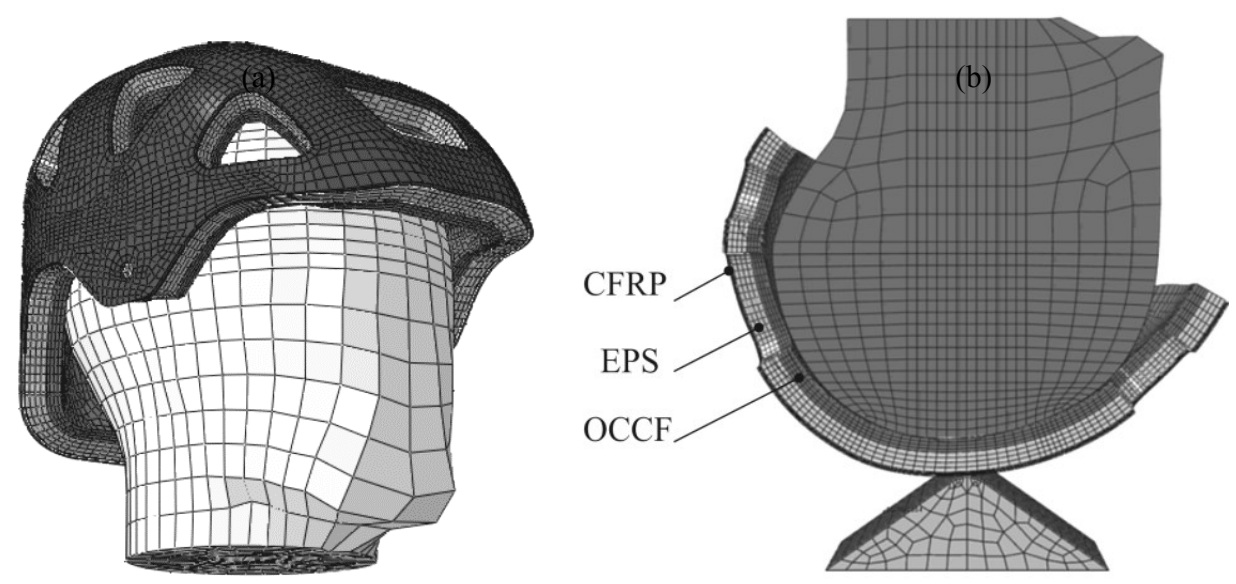

Fig. 2. FEM model of helmet (a), numerical simulation of one of helmet drop test according to ČSN EN 1078 (b)

Performed simulations were supplemented by the numerical drop test of a sample representing one cell of helmet foam liners. Two types of samples having different shapes of the interaction surface (see Fig. 3) were tested using a virtual flat impactor.

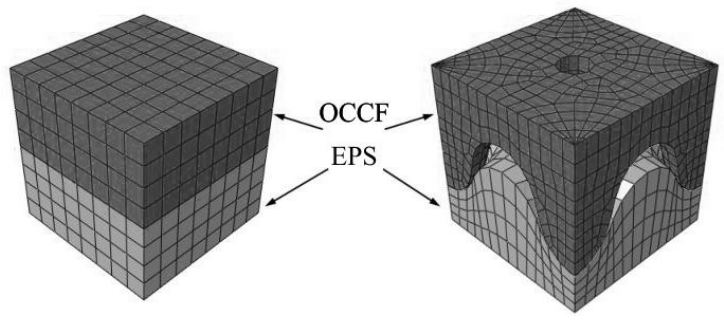

Fig. 3. "Plate" (left) and "conical" (right) design of liners 


\section{Results and discussion}

Different mechanical behaviour of the EPS and the OCCF is obvious from the compression test (Fig. 4(a)). The EPS absorbed a great amount of energy in first loading only. Size of the hysteresis loop in case of the second EPS loading was nearly negligible, which is in agreement with [7]. Size of the first loading hysteresis loop in case of the OCCF compression test was lesser, nevertheless, OCCF behaviour was almost identical in both loading cycles.

Average maximum forces measured in the drop test using the flat impactor are shown in Fig. 4(b). It is obvious that the value of the maximum force increased with the impact velocity more significantly in case of the OCCF.

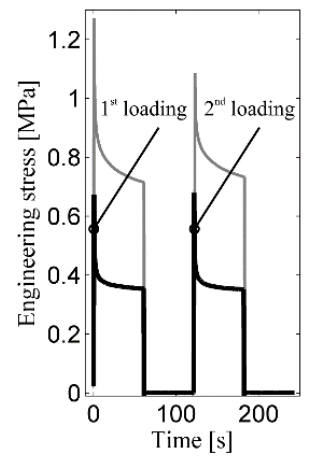

(a)

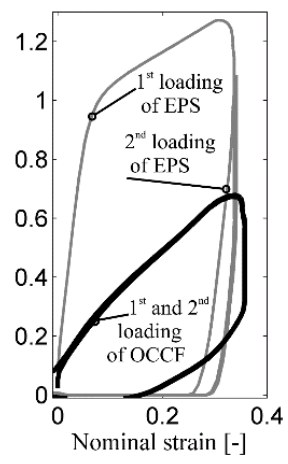

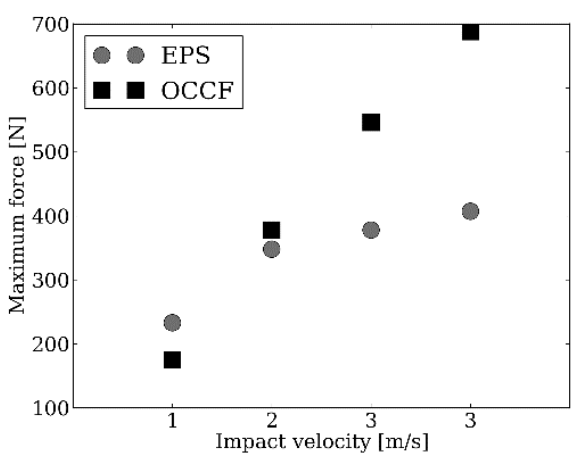

(b)

Fig. 4. Typical dependences of engineering stress on time and nominal strain in compression test (a), maximum forces measured in drop test using flat impactor (b)

Identified parameters of the material models are listed in Tables 1-2. The EPS parameters are: Poisson's ratio $v_{\mathrm{EPS}}$, Young's modulus $E_{\mathrm{EPS}}$, compression yield stress ratio $k$, initial yield stress $S_{0}$, yield stress $S_{1}$ corresponding to uniaxial plastic strain $\varepsilon_{\mathrm{p} 1}$, and yield stress $S_{2}$ corresponding to uniaxial plastic strain $\varepsilon_{\mathrm{p} 2}$. The OCCF parameters are: Poisson's ratio $v_{\mathrm{OCCF}}$, Young's modulus $E_{\mathrm{OCCF}}$, normalized model parameters $g_{1}, g_{2}$ and $g_{3}$, and relaxation times $\tau_{1}, \tau_{2}$ and $\tau_{3}$. It is obvious that the OCCF parameters differ based on the impact velocity (energy). Therefore, the experimental values were approximated as energy dependent functions in the numerical simulations. The comparison of the force response in case of the OCCF drop test using the spherical impactor for the impact velocity $v=4.5 \mathrm{~m} / \mathrm{s}$ is shown in Fig. 5.

Table 1. Identified parameters of EPS

\begin{tabular}{|c|c|c|c|c|c|c|c|c|}
\hline Param. & $\boldsymbol{v}_{\mathrm{EPS}}$ & $\boldsymbol{E}_{\mathbf{E P S}}$ & $\boldsymbol{k}$ & $\boldsymbol{S}_{\mathbf{0}}$ & $\boldsymbol{S}_{\mathbf{1}}$ & $\boldsymbol{\varepsilon}_{\mathbf{p} 1}$ & $\boldsymbol{S}_{\mathbf{2}}$ & $\boldsymbol{\varepsilon}_{\mathbf{p} 2}$ \\
\hline Units & - & $\mathrm{MPa}$ & - & $\mathrm{MPa}$ & $\mathrm{MPa}$ & - & $\mathrm{MPa}$ & - \\
\hline Value & 0.07 & 37.7 & 1.14 & 0.53 & 0.85 & 0.11 & 1.2 & 0.32 \\
\hline
\end{tabular}




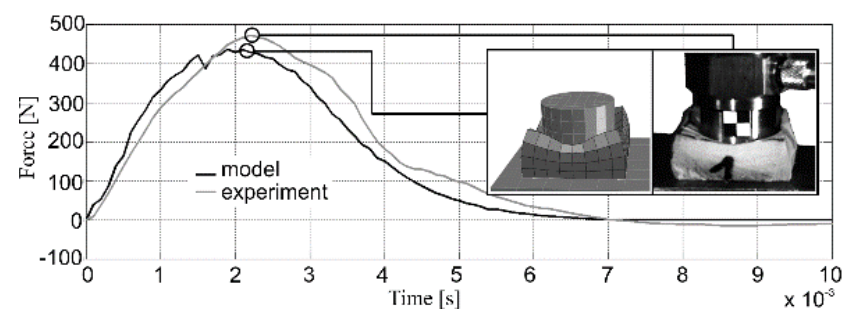

Fig. 5. OCCF drop test using spherical impactor for velocity of $4.5 \mathrm{~m} / \mathrm{s}$

Table 2. Identified parameters of OCCF

\begin{tabular}{|c|c|c|c|c|c|}
\hline \multirow{2}{*}{ Param. } & \multirow{2}{*}{ Units } & \multicolumn{5}{|c|}{$v[\mathbf{m} / \mathbf{s}]$} \\
\cline { 3 - 6 } & & $\mathbf{1}$ & $\mathbf{2}$ & $\mathbf{3}$ & $\mathbf{4}$ \\
\hline \hline$v_{\text {OCCF }}$ & - & \multicolumn{5}{|c|}{0.38} \\
\hline$E_{\text {OCCF }}$ & $\mathrm{MPa}$ & 1.99 & 0.69 & 0.62 & 0.55 \\
\hline$g_{1}$ & - & 0.67 & 0.54 & 0.56 & 0.65 \\
\hline$\tau_{1}$ & $\mu \mathrm{s}$ & 103 & 48 & 43 & 11 \\
\hline$g_{2}$ & - & 0.23 & 0.43 & 0.41 & 0.30 \\
\hline$\tau_{2}$ & $\mu \mathrm{s}$ & 242 & 113 & 101 & 25 \\
\hline$g_{3}$ & - & 0.046 & 0.023 & 0.023 & 0.043 \\
\hline$\tau_{3}$ & $\mu \mathrm{s}$ & 1693 & 791 & 708 & 204 \\
\hline
\end{tabular}

The numerical drop test shown that a significant weight save can be achieved using the "conical" design of the liner. Fig. 6 shows the comparison of the force impact response in case of the "plate" and the "conical" design of the liner. It is obvious that differences between maximum forces are not significant, however, the difference of weight is $20 \%$. Moreover, the "conical" design has lower stiffness in tangential direction, which is important for the brain protection [8].

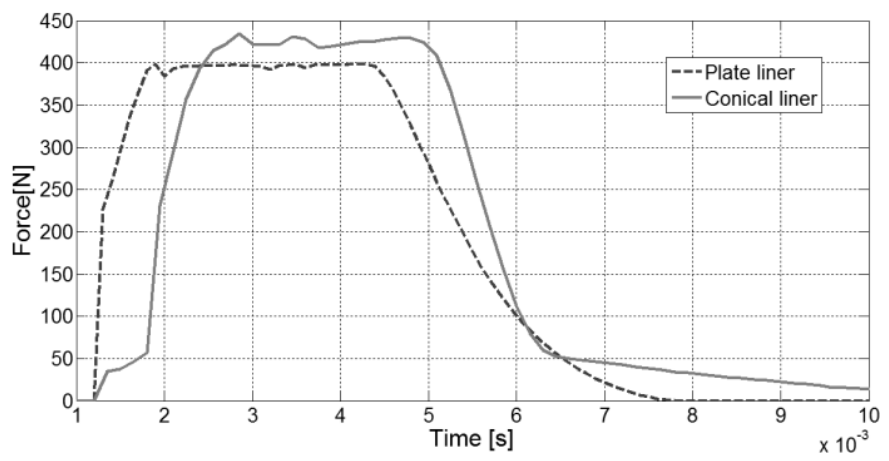

Fig. 6. Comparison of the plate and conical liners design in numerical drop test for velocity of $4 \mathrm{~m} / \mathrm{s}$

Numerical simulations of helmet drop tests according to standards ČSN EN 1078 and CSN EN 1385 showed that the thickness of the EPS layer must be at least $10 \mathrm{~mm}$ and the thickness of the OCCF layer must be at least $7 \mathrm{~mm}$ (for analysed type of helmet). 


\section{Conclusions}

The crushable foam material model and the Prony series were used to describe mechanical behaviour of the EPS and the OCCF, respectively. The parameters of the material models were identified, with the emphasis on the fitting of the drop tests. The "conical" design of the helmet liner allows weight savings of up to $20 \%$ and it is also more suitable because of its lower tangential stiffness. Based on the results of the numerical simulations, the thickness of the versatile helmet lining must be at least $17 \mathrm{~mm}$.

This publication was supported by the project LO1506 of the Czech Ministry of Education, Youth and Sports.

\section{References}

1. R. Hynek, Versatile Sport Helmet. (University of West Bohemia, Pilsen, 2014)

2. L. Cui, S. Kiernan, M.D. Gilchrist, Designing the energy absorption capacity of functionally graded foam materials. Materials Science and Engineering A 507, 215-225 (2009)

3. R. Kottner, T. Mandys, J. Barotšek, J. Kocáb, Impact force response of foams and rubbers. Proc. of the $54^{\text {th }}$ Conf. on Experimental Stress Analysis, Srní, 1-7 (2016)

4. W. Tanwongwan, J. Carmai, Finite element modelling of titanium foam behaviour for dental application. Proc. Of the World Congress on Engineering 2011, Vol. III, London, $1-6(2011)$

5. V.S. Deshpande, N.A. Fleck, Isotropic constitutive models for metallic foams. Journal of the Mechanics and Physics of Solids 48, 1253-1268 (2000)

6. J. Bergström, Mechanics of Solid Polymers. (Elsevier, San Diego, 2015)

7. F.M. Shuaeib, A.M.S. Hamouda, S.V. Wong, R.S. Radin Umar, M.M.H. Megat Ahmed, A new motorcycle helmet liner material: The finite element simulation and design of experiment optimization. Materials and Design 28, 182-195 (2007)

8. V.G. Ivancevic, New mechanics of traumatic brain injury. Cogn. Neurodyn 3, 281-293 (2009) 